\title{
Designing and Evaluating Casual Health Games for Children and Teenagers with Cancer
}

\author{
Kathrin Gerling, Alberto Fuchslocher, Ralf Schmidt, \\ Nicole Krämer, and Maic Masuch \\ University of Duisburg-Essen, Forsthausweg 2, \\ 47057 Duisburg, Germany \\ \{kathrin.gerling, alberto.fuchslocher, ralf.schmidt, \\ nicole.kraemer, maic.masuch\}@uni-due.de
}

\begin{abstract}
Because they offer an easy entry into play, casual games have become an increasingly popular leisure activity among children and teenagers, engaging particularly broad target audiences. In this paper, we present a casual game that addresses childhood cancer: Besides mere entertainment, a health game that focuses on cancer may serve as a clinical tool in order to teach children about the particularities of the disease and initiate discussion among cancer patients, their parents and medical staff. In this context, the results of an empirical study revealed a generally high acceptance of the health game among young patients, while parents and medical staff highlighted the educational potential of health games addressing cancer. Additionally, we discuss the challenges of evaluating digital games in a hospital setting which were revealed during the evaluation phase.
\end{abstract}

Keywords: Casual Games, Game Design, Games for Health, Serious Games, Childhood Cancer, Persuasive Games.

\section{Introduction}

Besides the persuasive potential of serious games which may be applied to encourage healthy behavior $[1,6]$, their benefits can be found beyond persuasion: Serious games generally offer the opportunity of raising awareness for difficult topics, for instance environmental or social issues and health-related topics such as disease treatment or prevention [30]. In the context of designing games for health, childhood cancer can be regarded as a particularly sensitive issue as it still presents a common cause of death among children and teenagers in industrialized countries [1, 35]. While the diagnosis severely influences regular family life, treatment is often painful and requires frequent periods of hospitalization and regular doctor's visits [10]. In addition, patients are faced with psycho-emotional problems, such as anxiety or depression [1, 23, 28]. Based on the patient's difficult situation, a need for health games dealing with their own medical condition has already been expressed [28]. In this context, games for health offer the potential of engaging patients and healthy children in play while simultaneously conveying information about cancer, but may not be fully accessible to players without prior gaming experience and persons suffering from treatment sideeffects. 
This issue may be addressed by introducing casual game design principles to health games: During the past years, casual games have become increasingly popular due to their flexibility and their usually well-accessible design [19]. Their popularity is also reflected by market research evidence, suggesting that such games offer the potential to reach particularly broad audiences [5]. In consequence, there have been attempts to integrate casual game elements into numerous game concepts, such as health games [16]. This is especially important as younger audiences are hard to reach through traditional communication channels [9] but may be addressed by digital games [25]. By this means, casual health games can be employed as an alternative approach to inform kids and teenagers about their medical condition and foster communication between patients and their friends, family as well as the medical staff.

In this paper, casual game design is discussed with a focus on the integration of serious game elements, particularly addressing games for health. Based on these considerations, the game prototype Cytarius was developed, which aims to illustrate cancer treatment and to convey information about the disease through its background story and game mechanics. The results of an empirical study in a hospital setting revealed that the game generally appeals to children and juvenile cancer patients. Furthermore, interviews with patients' parents show a general need for games designed for cancer patients and highlight the large potential of serious game concepts to initiate communication about the disease.

\section{Related Work}

Entertainment technology has previously been applied to ease everyday life of ill children, for instance through community-based approaches: Onko-Kids is a web portal specifically developed for children and teenagers diagnosed with cancer, providing information about cancer and offering didactical animated videos about the disease. STARBRIGHT World is a web-based community for chronically ill children that does not address a particular medical condition, but features different mini-games without explicitly referring to a specific disease. Its beneficial purpose is based on research results suggesting that patients who engage in online platforms show higher treatment compliance, experience less pain, and develop better coping strategies [12].

Also, various health games addressing patient needs and motivation are available, which can generally be categorized as games for learning, games as distraction or games as coach [33]. Currently, a wide range of games for health focuses on learning and distraction, frequently dealing with chronic conditions, for instance diabetes and asthma [4, 21] or severe illness such as cancer [17]. Furthermore, certain health games address the issue of disease prevention [9]. Popular examples of clinically tested and commercially available health games are Packy \& Marlon, a game which addresses diabetes [4], and Bronkie the Bronchiasaurus, which conveys information about asthma during play [21]. Another clinically evaluated game that directly focuses on cancer and which improves the patients' adherence to medication plans and perceived self-efficacy is the action game Re-Mission [17]: The player enters the game world as nanobot which fights the disease from within young patients' bodies. The game aims to convey basic information about common cancer symptoms and treatment strategies through game mechanics, e.g. enemy and weapon design. Also, 
more simplistic games for kids and teenagers with cancer have been designed, such as Ben's Game [2] and Onko-Ocean [23]. However, only few casual games focusing on health issues with a solid theoretical background as well as empirical research are available. Yet, the results of a preliminary study examining effects of commercially available casual games suggest that such games may foster the emotional well-being of players and lead to a reduction of depression symptoms [26] which is promising in the context of designing positive gaming experiences for kids with cancer. Also, due to their simplistic nature, casual games may be especially appropriate for diverse audiences, with special needs. To examine this issue, the following section discusses common design principles and the integration of serious elements into casual games.

\section{Casual Game Design}

Casual games represent an approach of designing digital games for large user groups and claim to be suitable for all audiences due to simplistic game concepts and interaction paradigms [14, 20, 32]. Recent studies report that more than 200 million persons regularly play web-based casual games, which highlights the immense popularity of such games and their potential of reaching broad target groups [5].

\subsection{Characteristic Features of Casual Games}

A review of existing work examining the most important features of casual games revealed five main areas which are characteristic for casual games and which are expected to have largely contributed to their success.

On a formal level, usability and accessibility (cf. [14, 19]) represent important aspects of casual game design as they significantly contribute to the user's easy entry into play [32]. In this context, it is important to provide simple controls [18] which are accessible to novice players without prior gaming experience to ease their adaption to interaction paradigms and common game metaphors [13]. This corresponds with the idea of providing comprehensive game play and mechanics in order to create an adequate learning curve $[13,18]$. This is achieved by implementing simplistic game features which enable the player to easily progress through the game [13]. Furthermore, clear rules and objectives within the game as well as transparent challenges are important factors which contribute to the learnability of casual games $[13,32]$. Also, positive feedback and an overall high game responsiveness are important features of casual games. First, clear feedback contributes to the player's efforts of learning the game [13]. Second, fast rewards, an adequate level of difficulty and the abstinence of player punishment support the player's motivation of engaging in play $[14,18]$ accounting for the overall juiciness of the player's individual gaming experience [14]. The integration of the aforementioned features contributes to another important aspect of casual game design, namely accounting for a certain degree of flexibility [19]. By providing flexible gameplay, gaming sessions can easily be adapted to the user's way of life, which enables players to engage with casual games regardless of other commitments [32]. Research results suggest that the support of short play sessions and a high degree of interruptibility are crucial in this context [14, 19]. Finally, casual games have to provide an appealing narrative in terms of being 
relevant and thus interesting for large audiences [18]. This can be achieved by integrating topics evolving around everyday life [32] and considering the popularity of contents in order to raise interest of large groups of people [19]. Providing an appealing background story is also important because the game topic is often immediately visible to prospective players. Hence, fiction represents a key factor when choosing or discarding a game prior to entering play [14].

In order to draw conclusions regarding the design of health games, the following section discusses the aforementioned features with a focus on designing casual games for children and teenagers diagnosed with cancer.

\subsection{Designing Casual Games for Cancer Patients}

Health games are usually regarded as a subset of serious games, which are referred to as games with a purpose beyond mere entertainment [22, 34] aiming to convey knowledge and educate the user [25]. In the context of designing games for health, serious games aim to foster healthy behavior and to inform players, or to improve the player's condition by strengthening self esteem and perceived self-efficacy $[11,17]$.

Regarding the design of casual games with a serious background, an integration of serious game elements such as disease-related information as well as main features of casual games, such as accessibility, flexibility and learnability has been claimed to be advantageous [34] and can also be applied to the design of games for health.

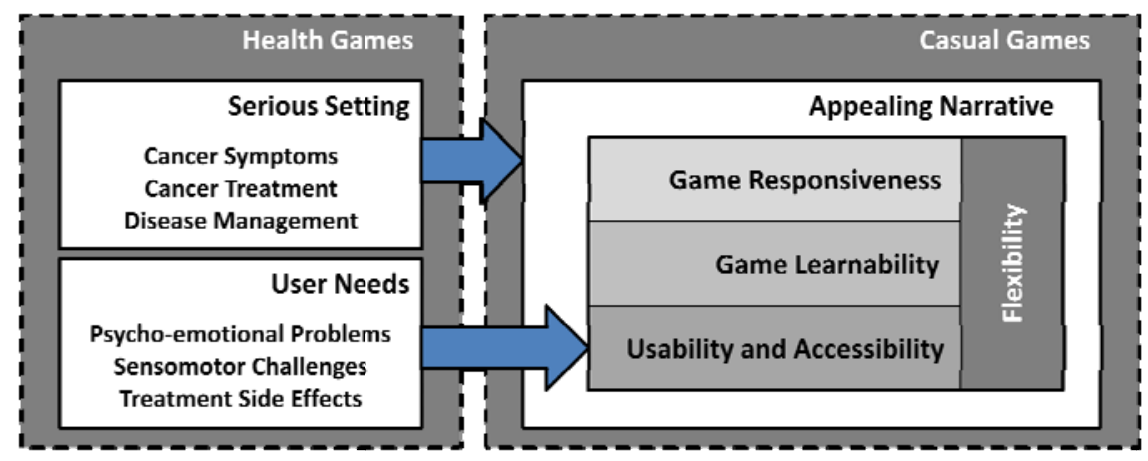

Fig. 1. The integration of serious game elements affects the design of casual games on two levels: The background story needs to be adjusted to a serious setting, and user needs have to be considered when designing core game mechanics

When designing casual games for children and teenagers with cancer, the possible impact of the disease on the emotional well-being as well as cognitive and physical abilities of juvenile cancer patients has to be considered [31]. First of all, periods of hospitalization and frequent doctor's visits require flexible games which can either be played at home or in a mobile context. Second, it is important to consider the impact of medication side effects if children are undergoing treatment, meaning that players may be weakened by medication side effects such as nausea or pain, thus game usability and accessibility as well as a high level of interruptibility must be considered. Third, providing an appealing narrative which frames the gaming 
experience is necessary to raise prospective players' interest and to encourage existing users to return to play regardless of their medical condition. In this context, diseaserelated information has to be integrated carefully as research results regarding the implementation of explicit or implicit disease references are contradictory $[7,8]$.

To conclude, the integration of serious game elements to create casual games for health influences the game design process in two ways (cf. Figure 1): On the one hand, the serious setting of the game has to be combined into an appealing narrative, which may be challenging if the original topic holds a negative connotation. On the other hand, additional user needs derived from the specific situation of the target audience have to be considered, therefore influencing the design of core features of casual games.

\section{Game Concept: Cytarius}

Based on the aforementioned considerations regarding casual game design as well as special requirements of children and teenagers with cancer, the game concept Cytarius was developed and implemented as a playable prototype. The game is set in a science fiction narrative and integrates different types of cancer and treatment options as game mechanics. Thereby, it playfully tries to inform the user about basic aspects of the disease and common treatment options.

\subsection{Core Features}

The serious setting of the game is closely related with its narrative and is represented by the background story and game mechanics which playfully convey information about the cause and treatment of cancer. The background story is set in space and evolves around the four planets Haima, Enképhalon, Blaston and Cytarius. While the inhabitants of Enképhalon and Haima live in peace, inhabitants of Blaston have been excluded from the intergalactic community due to selfish behavior. To take revenge, they try to infiltrate the community by rapidly reproducing themselves and gaining control over the other planets. To defeat the intruders, the inhabitants of Cytarius genetically engineered Cytowarriors who are lead by the player - try to defend the two peaceful planets. The core mechanic of the game is the strategic application of different types of warriors, which can be chosen through an in-game menu and placed in the game area by pointing and clicking at the desired spot (cf. Figure 2). Once a warrior is added to the game, it automatically executes its characteristic behavior for a certain amount of time, e.g. it destroys all other creatures within a certain radius.

To increase the usability and accessibility of Cytarius, basic interaction paradigms and a simplistic user interface were implemented. The game requires mouse input only, which allows the player to focus on one input device. Well-readable fonts as well as high-contrast graphics were used for the graphical user interface which can easily read by patients even when playing the game from their hospital beds. During game play, a limited amount of actions is available to the player. This also contributes to the game learnability, which is achieved by a gradually rising level of difficulty as the player advances through the game, and the availability of different in-game speed settings. Furthermore, a comic-based tutorial introduces the most important game 
elements to the player. A high game responsiveness is achieved by providing immediate visual and auditive feedback to the player's actions, e.g. the successful application of Cytowarriors is visualized by characteristic animations and sounds.

The flexibility of the game is ensured by offering an in-game menu which allows the player to immediately pause the game. Furthermore, it is possible to save individual progress and return to play at a later point of time.

\subsection{Game Play}

In Cytarius, the player is offered the role of a strategic commander. To master the game, it is crucial to coordinate the activities of Cytowarriors which are equipped with different weapons which were designed based on different cancer treatments. Thereby, it is possible to control the activities of Blasts and protect the inhabitants of Haima and Enképhalon.

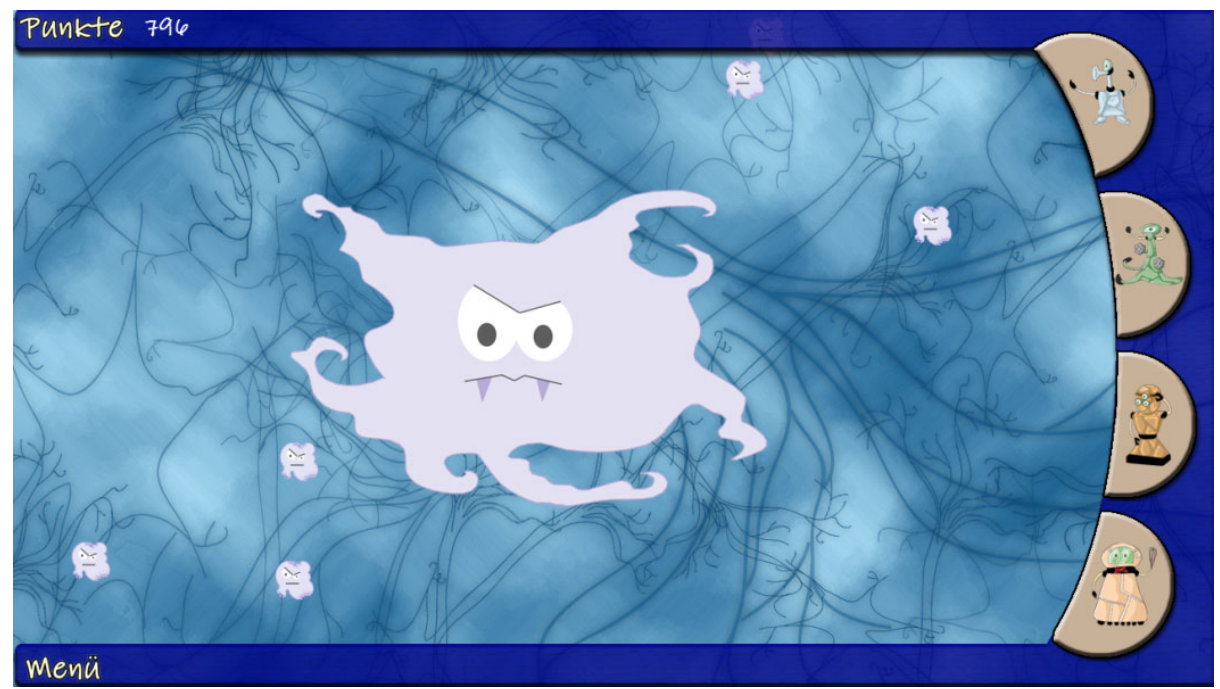

Fig. 2. Cytarius playfully conveys information on different types of cancer, for instance tumors and the development of metastases

Planet Haima: Leukemia. The background story and graphical design of the first level which is set on Haima are based on an analogy to leukemia: To win the level, the player has to fight Blasts which have infiltrated the planet. Their unregulated reproduction leads to the extinction of native inhabitants, Erys and Leucos. In order to save the planet, different Cytowarriors with features resembling core aspects of chemotherapy have to be applied to guarantee the survival of a minimum amount of Erys and Leucos while reducing the number of Blasts.

Planet Enkephalon: Tumors. On Enképhalon, the player is faced with a giant creature resembling a metastasizing tumor (cf. Figure 2), which has to be fought in order to allow native inhabitants to return to the planet. In this level, Cytowarriors 
may be applied to execute irradiation in strategically important areas and to reduce the amount of metastases.

The overall goal of Cytarius is to offer a first insight into processes related to cancer and its treatment options by engaging users with cancer-related game content. Thereby, the game aims to convey knowledge about the disease to inform juvenile cancer patients and to lay a foundation for future discussions of their medical condition with their parents and medical staff. Also, the game may be used to inform healthy juveniles about childhood cancer. Based on the concept described within this section, a playable prototype was implemented using Microsoft Game Studio. It was tested in the context of an evaluation with juvenile cancer patients, their parents and medical staff.

\section{Evaluation}

In order to integrate juvenile cancer patients in the further development process of Cytarius and to derive additional information regarding the design of casual games with a serious background, the evaluation was realized in two steps. First, children and teenagers with cancer were introduced to the game Cytarius and asked to fill out a questionnaire addressing the usability and accessibility of the game as well as their gaming experience. Second, qualitative interviews were conducted in order to gain further insight into players' perception of the game and to explore opinions of parents and medical staff.

\subsection{Participants and Procedure}

To evaluate the accessibility, acceptance and user experience evoked by the game prototype, an empirical study was conducted. Participants were recruited at the University Hospital Essen and the Hannover Medical School, Germany. Both stationary and ambulant patients took part in the study. The experimental procedure consisted of playing the game during a single session for fifteen minutes and answering a questionnaire. In the first part of the questionnaire, participants described their media usage and social network behavior. Subsequently, after having played the game for a 15-minute interval, game accessibility and player experience were assessed based on the Game Experience Questionnaire (GEQ [14]). Furthermore, participants were asked about their physical and psychic health condition (KINDL [26]) in order to further examine whether the casual game suited the needs and restrictions of the target audience. All variables were assessed on a 5-point rating scale. Additional to those playtesting elements of the evaluation, qualitative interviews with nursing staff and parents were conducted to examine their attitudes in regard to health games.

23 children and juveniles with cancer (17 boys and 6 girls) were able to take part in the study. They age range of study participants was between 7 and $19(\mathrm{M}=13.39$; $\mathrm{SD}=3.34$ ) years. Almost $4 / 5$ of the sample $(78 \%)$ reported having an own personal computer or laptop and $91 \%$ of the sample described to have experience with computer games while no specific genre preferences were found. Over half of the sample (12 of 23 participants) reported being registered in a social network site such as SchülerVZ or facebook.

The qualitative data consists of ten interviews with parents of children and juveniles diagnosed with cancer. Additionally, five interviews with clinical staff of 
the oncology departments of the hospitals in Hannover and Essen were conducted. The interviews were realized in order to explore the perception of parents and medical staff regarding their acceptance of digital entertainment for children diagnosed with cancer as well as future design opportunities.

\subsection{Results}

Quantitative Evaluation. Study results reveal that Cytarius was positively evaluated by study participants $(\mathrm{M}=3.71 ; \mathrm{SD}=1.05)$, suggesting that juveniles generally enjoyed playing the game. This notion is reinforced by the observed GEQ positive and negative affective reactions. Therefore, results of a t-test for dependent sample revealed that children experienced significantly more positive $(\mathrm{M}=3.26 ; \mathrm{SD}=1.25)$ than negative emotional affective reactions $(\mathrm{M}=1.36 ; \mathrm{SD}=.77)$ during gameplay $\left(\mathrm{t}_{(22)}=5.36 ; \mathrm{p}=.000\right)$. Furthermore study participants seemed to specially like the game story assessed by the immersion dimension of the GEQ $(\mathrm{M}=3.45$; $\mathrm{SD}=1.33)$. However participants only experienced an average flow level $(\mathrm{M}=2.8 ; 1.15)$ as a consequence of playing the game.

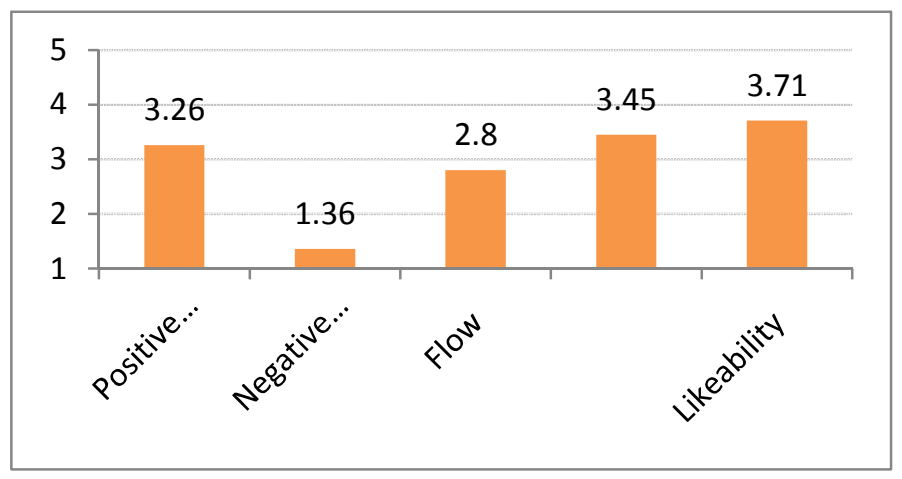

Fig. 3. Mean results of the main dimensions of the Game Experience Questionnaire as well as game likability show a generally positive gaming experience among the participants of the evaluation

In a further analysis, the influence of participants' demographic characteristics on the evaluation results was analyzed in order to investigate game adequacy for different subgroups. The results of a correlation analysis revealed a significant negative correlation between participants' age and game likeability $(\mathrm{r}=-.572 ; \mathrm{p}=$ .004 ), suggesting that especially young participants liked the game. In congruence with this finding, the participants' age was also found to be negative interrelated with the story evaluation $(\mathrm{r}=-.524 ; \mathrm{p}=.010)$ and flow $(\mathrm{r}=-.486 ; \mathrm{p}=.019)$ dimension of the game experience questionnaire. The results suggest that young participants liked the game $(\mathrm{M}=4.19 ; \mathrm{SD}=.936)$ and enjoyed the story $(\mathrm{M}=4.04 ; \mathrm{SD}=1.17)$ more than older participants $(\mathrm{M}=3.18 ; \mathrm{SD}=.947 ; \mathrm{M}=2.81 ; \mathrm{SD}=1.25$, respectively). No significant interrelations between participant's age and positive $(r=-.085 ; \mathrm{p}=.699)$ or negative emotional reactions $(r=-.076 ; \mathrm{p}=.729)$ were found. 
The assessment of patients' health condition (KINDL) was necessary in order to analyze if a patient's physical or psychological restrains affected game enjoyment. In general, most participants reported having no difficulties in operating a mouse $(92 \%)$ or a keyboard $(88 \%)$. The results of the KINDL questionnaire revealed that the participants' physical (60.5 of 100 points) and psychic (70 of 100 points) health condition was relatively normal in comparison to a healthy juvenile population (75 and 83 points, respectively). However, a correlation analysis revealed marginal interrelations between the participants' physical health condition and the GEQ dimensions game flow $(\mathrm{r}=-.380 ; \mathrm{p}=081)$ and positive affect $(\mathrm{r}=-.379 ; \mathrm{p}=082)$, suggesting that decrements in the patients' physical health also influence the quality of their individual game experience.

Qualitative Evaluation. When questioned about the use of digital media among their children, six out of ten parents reported to restrict their children's access to the computer. However, a majority of eight out of ten parents agreed that playing games specifically designed for cancer patients would be beneficial in terms of learning about the disease and developing adequate coping strategies. The most frequent argument for a cancer related health game was augmenting their children's understanding of their medical condition (4 of 8 parents). Furthermore, parents claimed that games might be used to initiate discussions about the disease and generally foster an optimistic, open approach to dealing with cancer. In congruence to these assertions, medical staff interviews unanimously revealed the need for a health game that entertains and interconnects juveniles while learning about their medical condition. Despite the numerous entertainment activities offered in the oncologic stations, medical staff reported that only few patients take part on it due to treatment side effects: Their preferred activities were playing video games and watching TV.

\subsection{Discussion}

Results suggest that participants generally enjoyed playing the game. This is reflected on positive affective reactions elicited by playing the game. Moreover, especially young patients liked the game and its story. However no significant difference on flow experience was found between young and older patients, suggesting a comparable gaming experience among the participants. Furthermore, interviews with parents and medical stuff revealed an existing demand for a health oriented casual game, due to children's main interest regarding gaming and the necessity of fostering children's communication about their medical condition.

On a general level, evaluating Cytarius in a hospital context was challenging as certain restrictions regarding the procedure of the evaluation were present which partially derived from the participants' condition as well as the hospital setting. First of all, the patients' condition did not always allow them to take part in the study without interruptions. Furthermore, despite the general interest to take part in the study, some patients were simply not in the psychical or physical condition to participate. Second, stationary patients are usually placed in shared bedrooms, thus the evaluation often could not take place in a quiet environment as many patients were too weak to leave their beds. Finally, appointments for the evaluation had to be treated with a high flexibility as the patient's condition often varied on a daily basis depending on treatment routines. 


\section{Conclusion and Future Work}

In this paper, a theoretical analysis of casual games with a focus on creating casual health games through the integration of serious game elements is introduced. Based on these theoretical considerations, the game prototype Cytarius is presented, which addresses the issue of childhood cancer. An evaluation of a playable game prototype for children and teenagers with cancer suggests that juvenile cancer patients generally enjoy engaging with such a game. Furthermore the potential of casual game concepts to initiate further discussions about their medical condition is highlighted, an idea which is supported by results of interviews with parents and medical staff.

The development of the game prototype Cytarius has shown that serious game elements may easily be integrated into casual game concepts. Regarding the acceptability and accessibility of the game, the results of the evaluation suggest that Cytarius provides a generally enjoyable gaming experience for kids and teenagers with cancer. The results show that the game is suited to be played in a hospital setting, and that it is widely accessible to its players despite cognitive and physical consequences of the disease. However, age-related differences in the participants' ratings also reveal that the current game story and the graphical design may not be equally appealing for all audiences and should be subject to future design considerations. In order to draw conclusions regarding possible positive effects of regularly engaging with casual games for health on cancer patient's medical condition (similar to those observed among cancer patients playing Re-Mission [17]), further research is necessary.

Future work includes a longitudinal clinical evaluation of Cytarius featuring a larger sample size and additional game elements. Also, it is planned to extend the existing game prototype into a fully playable casual game offering connectivity to social network websites, e.g. facebook, and featuring social game mechanics such as the integration of friends into play. Thereby, we expect to reach a broader audience including healthy adolescents and inform them about the difficult situation of peers who have been diagnosed with cancer.

Acknowledgments. We would like to thank Ramsi Heni for the dedicated work on his Bachelor's thesis, which largely contributed to the development of Cytarius. Furthermore, thanks and our best wishes for the future go to all young patients and their families who participated in the evaluation. Also, we would like to thank PD Dr. Lorenz Grigull at the Department of Paediatric Haematology and Oncology at Hannover Medical School and Dr. Oliver Basu at the Medical Department of the University of Duisburg-Essen for their extensive support throughout the evaluation.

\section{References}

1. Beckmann, I.-A.: Krebs im Kindesalter. Deutsche Krebshilfe e.V., Bonn (2007)

2. Ben's Game, http: / / www. sfwish. org / site/pp.asp? c=bdJLITMAE\&b=81924

3. Bogost, I.: Persuasive Games. The MIT Press, Cambridge (2007)

4. Brown, S.J., Lieberman, D.A., Gemeny, B.A., Fan, Y.C., Wilson, D.M., Pasta, D.J.: Educational video game for juvenile diabetes: Results of a controlled trial. Medical Informatics 22(1), 77-89 (1997) 
5. Casual Games Association,

http: //www. casualgamesassociation.org/pdf/

2007_CasualGamesMarketReport.pdf

6. Fogg, B.J.: Persuasive Technology: Using Computers to Change What We Think and Do. Morgan Kaufmann Publishers, San Francisco (2003)

7. Fuchslocher, A., Gerling, K., Masuch, M., Krämer, N.: Evaluating Social Games for Kids and Teenagers Diagnosed With Cancer. In: Proceedings of SeGAH 2011, Braga, Portugal (2011)

8. Fuchslocher, A., Niesenhaus, J., Krämer, N.: Serious Games for Health: An Empirical Study of the Game, Balance' for Teenagers With Diabetes Mellitus. In: Entertainment Computing (in press)

9. Gerling, K., Klauser, M., Masuch, M.: Serious Interface Design for Dental Health: WiiMote-based Tangible Interaction for School Children. In: Proceedings of Entertainment Interfaces 2010, Duisburg, Germany (2010)

10. Göbel, U.: Leukämien bei Kindern und Jugendlichen: Vom Symptom zur Diagnose. Monatsschrift Kinderheilkunde 151(2), 131-137 (2003)

11. Griffiths, M.: The Therapeutic Value of Video Games. In: Raessens, J., Goldstein, J. (eds.) Handbook of Computer Game Studies. The MIT Press, Cambridge (2005)

12. Holden, G., Bearison, D.J., Rode, D.C., Fishman-Kapiloff, M., Rosenberg, G., Onghena, P.: Pediatric Pain and Anxiety: A Meta-Analysis of Outcomes for a Behavioral Telehealth Intervention. Research on Social Work Practice 13(6), 693-704 (2003)

13. IGDA Casual Games SIG, 2008-2009 Casual Games White Paper, http: / /www.igda.org/casual-games/casual-game-white-papers

14. Ijsselsteijn, W.A., de Kort, Y.A.W., Poels, K.: The Game Experience Questionnaire: Development of a self-report measure to assess the psychological impact of digital games (in preparation)

15. Juul, J.: A Casual Revolution. The MIT Press, Cambridge (2010)

16. Kato, P.M.: Video Games in Health Care: Closing the Gap. Review of General Psychology 14(2), 113-121 (2010)

17. Kato, P.M., Cole, S.W., Bradlyn, A.S., Pollock, B.H.: A Video Game Improves Behavioral Outcomes in Adolescents and Young Adults With Cancer: A Randomized Trial. Pediatrics 122(2), 305-317 (2008)

18. Kuittinen, J., Kultima, A., Niemelä, J., Paavilainen, J.: Casual Games Discussion. In: Proceedings of FuturePlay 2007, Toronto, Canada (2007)

19. Kultima, A.: Casual Design Values. In: Proceedings of MindTrek 2009, Tampere, Finland (2009)

20. Kultima, A., Stenros, J.: Designing Games for Everyone: The Expanded Game Experience Model. In: Proceedings of FuturePlay 2010, Vancouver, Canada (2010)

21. Lieberman, D.: The Researcher's Role in the Design of Children's Media and Technology. In: Druin, A. (ed.) The Design of Children's Technology. Morgan Kaufmann Publishers, San Francisco (1999)

22. Michael, D., Chen, S.: Serious Games. Games that Educate, Train, and Inform. Thomson Course Technology, Boston (2006)

23. Moody, K., Meyer, M., Mancuso, C.A., Charlson, M., Robbins, L.: Exploring concerns of children with cancer. Supportive Care in Cancer 14(9), 960-966 (2006)

24. Onko-Ocean, http://www. onko-kids.de/kidsfuerkids/ocean.htm

25. Prensky, M.: Digital Game-Based Learning. Paragon House, St. Paul (2007)

26. Ravens-Sieberer, U., Bullinger, M.: Assessing health related quality of life in chronically ill children with the German KINDL: First psychometric andcontent-analyticresults. Quality of Life Research 7, 399-407 (1998) 
27. Russoniello, C.V., Fish, M., O’Brien, K., Pougatchev, V., Zirnov, E.: The Efficacy of Prescribed Casual Video Games in Reducing Clinical Depression and Anxiety. PopCap Games,

http: / / www. ecu . edu/cs-hhp/rcls/biofeedback/upload/

Updated_Efficacy-of-CVGs-and-depression-study0200811.pptx

28. Sanger, M.S., Copeland, D.R., Davidson, E.R.: Psychosocial Adjustment Among Pediatric Cancer Patients: A Multidimensional Assessment. Journal of Pediatric Psychology 16(4), 463-474 (1991)

29. Schiffman, J.D., Csongradi, E., Suzuki, L.K.: Internet Use Among Adolescent and Young Adults (AYA) With Cancer. Pediatric Blood and Cancer 51, 410-415 (2008)

30. Suhonen, K., Väätäjä, H., Virtanen, T., Raisamo, R.: Seriously Fun - Exploring How to Combine Promoting Health Awareness and Engaging Gameplay. In: Proceedings of MindTrek 2008, Tampere, Finland (2008)

31. Suzuki, L.K., Kato, P.M.: Psychosocial support for patients in pediatric oncology: The influences of parents, schools, peers, and technology. Journal of Pediatric Oncology Nursing 20(4), 159-174 (2003)

32. Trefry, G.: Casual Game Design: Designing Play for the Gamer in All of Us. Morgan Kaufmann Publishers, Burlington (2010)

33. Watters, C., Oore, S., Shepherd, M., Abouzied, A., Cox, A., Kellar, M., Kharrazi, H., Liu, F., Otley, A.: Extending the Use of Games in Health Care. In: Proceedings of the 39th Annual Hawaii International Conference on System Sciences, Kauai, Hawaii (2007)

34. White, K.: Casual Games Meet Serious Games. In: IGDA 2008-2009 Casual Games White Paper, http: / /www. igda.org/casual-games / casual-game-white-papers

35. World Health Organization and International Union Against Cancer, Global Action Against Cancer, http: / /www . who.int/cancer/media/en/

GlobalActionCancerEnglfull.paf 